\title{
Low-dose Quetiapine-induced Syndrome of Inappropriate Antidiuretic Hormone in a Patient with Traumatic Brain Syndrome
}

\author{
Sang-Gu Kang, Seo-Hyeon Choi, Hee-Yun Kim, Hye-Young Kim, Jae-Nam Bae, Jung-Sub Lee, Won-Hyoung Kim \\ Department of Psychiatry, Inha University Hospital, Incheon, Korea
}

\begin{abstract}
Syndrome of inappropriate antidiuretic hormone secretion (SIADH) is characterized by hyponatremia, low serum osmolality, and clinical euvolemia in the absence of diuretic medication. And the causes of SIADH are various, antipsychotic agents and traumatic brain injury (TBI) are well known. Quetiapine is often chosen to manage the maladaptive behavior of patients with post-TBI. Although a previous study reported that quetiapine doses ranging from 25 to 300 mg were effective and tolerable, the symptoms of the patient might be aggravated. The symptoms of TBI such as nausea, malaise, headache, lethargy, and mild cognitive deficits are similar to those of SIADH. So the differentiation between SIADH and TBI may be difficult. This paper reports a case of SIADH in a patient with a TBI after using a small dose of 25 to $50 \mathrm{mg}$ quetiapine.
\end{abstract}

KEY WORDS: Inappropriate ADH syndrome; Traumatic brain injury; Quetiapine.

\section{INTRODUCTION}

Inappropriate antidiuretic hormone (SIADH) syndrome is the most frequent cause of hyponatremia and has many causes. Among them, antipsychotic drugs and traumatic brain injury (TBI) are common causes of SIADH [1]. A previous case-control study reported the association between antipsychotic drugs and hyponatremia or SIADH (adjusted rate of returm, 1.58; 95\% confidence interval, 1.46-1.70) [2].

Antipsychotics, such as quetiapine, are often chosen to manage the maladaptive behavior of patients with post-TBI, such as irritability, aggression [3,4]. A previous study reported that quetiapine doses ranging from 25 to $300 \mathrm{mg}$ daily were effective and tolerable for reducing the impulsive aggression and irritability induced by TBI [3]. In the present case, SIADH was found in a patient with TBI after using a small dose of 25 to $50 \mathrm{mg}$ quetiapine.

Received: April 30, 2018 / Revised: July 17, 2018

Accepted: August 8, 2018

Address for correspondence: Won-Hyoung Kim

Department and Research Institute of Psychiatry, Inha University College of Medicine, 27 Inhang-ro, Jung-gu, Incheon 22332, Korea E-mail: ckgodman@hanmail.net

ORCID: https://orcid.org/0000-0002-6650-3685

\section{CASE}

A 49-year-old male patient with a TBI was admitted to the Department of Psychiatrics with insomnia and violent behavior. Two months ago, he had subdural hemorrhage after a falling down the stairs under the influence of alcohol. When he was brought to the Emergency department, he appeared loss of consciousness, disorientation and confusion. Since the history of trauma, he showed left side weakness as well as cognitive impairment and violent behavior and he also had intermittent generalized tonic-clonic seizure. He had been given levetiracetam $3,000 \mathrm{mg}$ a day due to a history of seizure and memantine $20 \mathrm{mg}$ a day under the diagnosis of the major neruocognitive disorder due to TBI. A recent report examined memantine in patients with moderate TBI and concluded that memantine reduced serum levels of neuron-specific enolase, a marker of neuronal damage [5].

After admitted to the hospital, he showed no signs of dry mouth symptoms and denied the presence or prior history of excessive water drinking. Quetiapine was used to manage the sleep and behavior problem $(25 \mathrm{mg}$ on hospital days 1 and 2, $50 \mathrm{mg}$ on the next seven days, orally at night).

The patient was normotensive and his blood pressure

(c) This is an Open-Access article distributed under the terms of the Creative Commons Attribution Non-Commercial License (http://creativecommons.org/licenses/by-nc/4.0) which permits unrestricted non-commercial use, distribution, and reproduction in any medium, provided the original work is properly cited. 
was 121/78 mmHg. A physical examination did not reveal any abnormal findings (e.g., peripheral edema). His electrolyte laboratory findings upon admission were as follows: serum sodium concentration $137 \mathrm{mEq} / \mathrm{L}$ (133-145 $\mathrm{mEq} / \mathrm{L})$, serum osmolarity $268 \mathrm{mOsm} / \mathrm{L} \quad(275-295$ $\mathrm{mOsm} / \mathrm{L})$, urine sodium concentration $29.9 \mathrm{mEq} / \mathrm{L}(<20$ $\mathrm{mEq} / \mathrm{L}$ ), and urine osmolarity $504 \mathrm{mOsm} / \mathrm{kg}$, several laboratory tests were conducted to examine the patient's condition. The results revealed a normal renal, liver, and thyroid function as well as hormone levels within the normal limits.

After using a small dose of quetiapine, the patient experienced aggravated sleep problems, irritability, aggression, and hyperactive delirium and the patient's serum sodium level decreased to $126 \mathrm{mEq} / \mathrm{L}$ with a serum osmolarity of $262 \mathrm{mOsm} / \mathrm{kg}$. The urine sodium level was 148 $\mathrm{mEq} / \mathrm{L}$ and the urine concentration was $504 \mathrm{mOsm} / \mathrm{kg}$. A physical examination showed no sign of peripheral edema. In terms of these findings, a diagnosis of SIADH was established according to the criteria described by Bartter and Schwartz [6]. During his hospitalization, a complete diagnostic work-up was performed, including thorough laboratory testing, as well as the brain, chest, and abdomen computed tomography scans. The above diagnostic procedures excluded other factors as the potential causes of the syndrome, such as malignancies, infections, and stroke.

The initial management of the patient included the intravenous infusion of a $3 \%$ sodium chloride $500-\mathrm{ml}$ solution over 24 hours, the addition of 5 -g salt to his diet, quetiapine withdrawal, a water restriction of 1,000 $\mathrm{ml} /$ day, and furosemide $20 \mathrm{mg} /$ day. After two days, his serum sodium concentration and plasma osmolarity were maintained within the normal range. The patient's insomnia and violent behavior were controlled with mirtazapine $15 \mathrm{mg}$ and lorazepam $2 \mathrm{mg}$ injection. After decreasing 3\% sodium chloride and furosemide, the patent's electrolytes recovered to within the normal range. During the period of SIADH and after the end of SIADH, he kept taking memantine and levetiracetam.

\section{DISCUSSION}

The symptoms of SIADH are nausea, malaise, headache, lethargy, and mild cognitive deficits. In severe cases, the syndrome may cause seizure, delirium, and coma
[1]. The CNS pathology, such as TBI is associated with SIADH syndrome [1]. Differentiation between SIADH and TBI is difficult because of their similar neurological manifestations [7].

Acute hyponatremia caused by SIADH in the setting of a traumatic cerebral injury has a reported prevalence, ranging from $2.3 \%$ to $36.6 \%$, depending on the selection criteria, time of study, and follow-up period [8]. Among all etiologies, SIADH accounts for more than $90 \%$ of cases of post-TBI hyponatremia [9]. SIADH might be caused by central nervous system disorders because of the disorganized inputs from the osmoreceptor cells of the anterior hypothalamus and brainstem cardiovascular regulatory centers [10].

Quetiapine is well tolerated and effective in reducing aggression in nonpsychotic syndromes [4]. The administration of quetiapine at doses of 25 to $300 \mathrm{mg}$ daily is well-tolerated and leads to significant reductions in aggression and irritability [3]. A number of drugs are known to cause SIADH [5]. Conventional antipsychotics and atypical psychotics can be associated with the development of the syndrome [11]. Polypharmacy and the concomitant use of anti-psychotic medications may precipitate hyponatremia and/or SIADH [2]. Mannesse et al. [2] reported 631 cases of hyponatremia associated with anti-psychotics, of which 38 cases were secondary to quetiapine.

These results are different from previous cases. This paper describes a rare case of SIADH in a patient with post$\mathrm{TBI}$ that was precipitated by a low dose of quetiapine. When drug-induced SIADH occurs, rapid discontinuation of the suspected agent is required. Additional actions should be taken, such as water restriction, salt-added diet, and furosemide administration [12]. In the present case, the clinical manifestations of the patient suggested that SIADH could occur due to the administration of quetiapine. Laboratory tests were then carried out. On the other hand, the rapid correction of hyponatremia can result in central pontine myelinolysis, and irreversible neurological complications, such as spastic quadriparesis [13].

SIADH hyponatremia induced by quetiapine in a TBI patient is extremely uncommon, as concluded from previous reports. To the best of the authors' knowledge, there are few reports showing a temporal association of the dose and severity of hyponatremia [14]. A TBI patient developed severe hyponatremia with low dose quetiapine. 
Overall, psychiatrists should be aware of the rare, but severe adverse effects of low dose quetiapine. In addition, an electrolyte laboratory test is essential if symptoms, such as insomnia, irritability, and delirium, are detected in a patient taking quetiapine. Quetiapine must be considered as one of the causes of SIADH in patients with TBI.

The use of quetiapine, even in small doses, can lead to SIADH in patients with a TBI history. Before giving a prescription, it is necessary to determine the patient's history. In addition, regular electrolyte laboratory tests should be conducted on patients with TBI.

\section{Conflicts of Interest}

No potential conflict of interest relevant to this article was reported.

\section{Author Contributions}

Conceptualization: Won-Hyoung Kim and Sang-Gu Kang. Investigation: Sang-Gu Kang and Seo-Hyeon Choi. Resources: Won-Hyoung Kim and Sang-Gu Kang. Supervision: Hee-Yun Kim, Hye-Young Kim, Jae-Nam Bae, and Jung-Sub Lee. Writing-original draft: Sang-Gu Kang. Writing-review \& editing: Won-Hyoung Kim.

\section{ORCID}

Sang-Gu Kang Seo-Hyeon Choi Hee-Yun Kim Hye-Young Kim Jae-Nam Bae Jung-Sub Lee Won-Hyoung Kim https://orcid.org/0000-0003-0824-9022 https://orcid.org/0000-0002-2726-5610 https://orcid.org/0000-0002-6766-5969 https://orcid.org/0000-0002-2313-8892 https://orcid.org/0000-0002-5024-6231 https://orcid.org/0000-0001-5585-0334 https://orcid.org/0000-0002-6650-3685

\section{REFERENCES}

1. Ellison DH, Berl T. The syndrome of inappropriate antidiuresis. N Eng/ J Med 2007;356:2064-2072.

2. Mannesse CK, van Puijenbrobroek EP, Jansen PA, van Marum $\mathrm{RJ}$, Souverein PC, Egberts TC. Hyponatremia as an adverse drug reaction of antipsychotic drugs: a case-control study in VigiBase. Drug Saf 2010,33:569-578.

3. Kim E, Bijlani M. A pilot study of quetiapine treatment of aggression due to traumatic brain injury. I Neuropsychiatry Clin Neurosci 2006; 18:547-549.

4. Corson AH, Barkenbus JE, Posey DJ, Stigler KA, McDougle CJ. A retrospective analysis of quetiapine in the treatment of pervasive developmental disorders. I Clin Psychiatry 2004;65. 1531-1536.

5. Mokhtari M, Nayeb-Aghaei H, Kouchek M, Miri MM, Goharani R, Amoozandeh A, et al. Effect of memantine on serum levels of neuron-specific enolase and on the Glasgow Coma Scale in patients with moderate traumatic brain injury. J Clin Pharmacol 2018:58:42-47.

6. Bartter FC, Schwarz WB. The syndrome of inappropriate secretion of antidiuretic hormone. AmJMed 1967;42:790-806.

7. Chang $\mathrm{CH}$, Liao JJ, Chuang $\mathrm{CH}$, Lee CT. Recurrent hyponatremia after traumatic brain injury. Am J Med Sci 2008;335. 390-393.

8. Agha A, Sherlock M, Phillips J, Tormey W, Thompson CJ. The natural history of post-traumatic neurohypophysial dysfunction. Eur J Endocrinol 2005;152:371-377.

9. Agha A, Sherlock M, Thompson CJ. Post-traumatic hyponatraemia due to acute hypopituitarism. QJM 2005;98:463464.

10. Wong LL, Verbalis JG. Systemic diseases associated with disorders of water homeostasis. Endocrinol Metab Clin North Am 2002;31:121-140.

11. Meulendijks D, Mannesse CK, Jansen PA, van Marum RJ, Egberts TC. Antipsychotic-induced hyponatraemia: a systematic review of the published evidence. Drug Saf 2010;33:101114.

12. Ramos-Levi AM, Duran Rodriquez-Hervada A, Mendez-Bailon M, Marco-Martinez J. Drug-induced hyponatremia: an updated review. Minerva Endocrinol 2014;39:1-12.

13. Spasovski G, Vanholder R, Allolio B, Annane D, Ball S, Bichet $\mathrm{D}$, et al. Clinical practice guideline on diagnosis and treatment of hyponatraemia. Nephrol Dial Transplant 2014;29 Supp/ 2:i1-i39.

14. Atalay A, Turhan N, Aki OE. A challenging case of syndrome of inappropriate secretion of antidiuretic hormone in an elderly patient secondary to quetiapine. South Med J 2007;100: 832-833. 